\title{
Lycium barbarum Polysaccharide Antagonizes LPS-Induced Inflammation by Altering the Glycolysis and Differentiation of Macrophages by Triggering the Degradation of PKM2
}

\author{
Huan Ding, ${ }^{a}$ Jing-jing Wang, ${ }^{b}$ Xiao-Ya Zhang, ${ }^{a}$ Lei Yin, ${ }^{a}$ and Tao Feng $*, c$ \\ ${ }^{a}$ Intensive Care Unit (ICU), Department of Critical Care Unit, General Hospital of Ningxia Medical University; \\ Yinchuan, Ningxia 750004, China: ${ }^{b}$ Coronary Care Unit (CCU), Department of Cardiology, General Hospital of \\ Ningxia Medical University; Yinchuan, Ningxia 750004, China: and ${ }^{c}$ Intensive Care Unit (ICU), Department of \\ Critical Care Unit, Ningxia Third Hospital; Yinchuan, Ningxia 750011, China. \\ Received September 17, 2020; accepted December 22, 2020; advance publication released online December 26, 2020
}

\begin{abstract}
Lipopolysaccharide (LPS)-induced inflammation is the leading cause of multiple organ failure in sepsis. Pyruvate kinase 2 (PKM2) is a protein kinase and transcriptional coactivator that plays an important role in glycolysis. Recent studies have confirmed that glycolysis maintains the M1 differentiation and induces immune activation in macrophages. Lycium barbarum polysaccharide (LBP), the main bioactive component of Chinese wolfberry, suppresses glycolysis and inflammation. Here, RAW264.7 macrophages were treated with LBP for evaluating its effects against LPS-induced inflammation. The differentiation of M1/M2 macrophages was assessed by flow cytometry for assessing the cell surface markers, CD86 and CD206. The enrichment of hypoxia inducible factor (HIF)-1 $\alpha$ and ubiquitin in the PKM2 protein complex was determined by co-immunoprecipitation. LBP suppressed LPS-induced glycolysis, differentiation of M1 macrophages, and the production of interleukin (IL)-1 $\beta$, tumor necrosis factor (TNF)- $\alpha$, and high mobility group (HMG) 1 proteins. The suppressive effects of LBP were similar to those of PKM2 knockdown, but were abolished by the overexpression of PKM2. LPS elevated the mRNA and protein levels of PKM2. LBP reduced the LPSinduced expression of PKM2 protein, but had no effects on the expression of PKM2 mRNA. LPS inhibited the ubiquitination of PKM2, probably by downregulating the expression of ubiquitin ligases, including Nedd4L, Nedd4, and Gnb2. LBP interfered with the inhibition of PKM2 ubiquitination by upregulating the expression of Nedd4L, Nedd4, and Gnb2. In conclusion, LBP suppressed the LPS-induced inflammation by altering glycolysis and the M1 differentiation of macrophages. The effects of LBP were mediated by the downregulation of PKM2 via enhanced ubiquitination.
\end{abstract}

Key words Lycium barbarum polysaccharide; pyruvate kinase 2 (PKM2); inflammation; glycolysis; macrophage differentiation; ubiquitination

\section{INTRODUCTION}

Sepsis is a systemic immune disorder that is caused by infections. More than 50.90 million people are annually diagnosed with sepsis worldwide. ${ }^{1-3)}$ Sepsis can cause multiple organ failure, including lung, liver, and kidney failure, as well as cognitive impairment. ${ }^{4}$ Individuals with sepsis have a high mortality rate, and the mortality rate can be as high as a $40-50 \%$ following the development of septic shock. ${ }^{3}$ This is due to the fact that patients with septic shock suffer cardiovascular collapse and are often unresponsive to fluid resuscitation and treatment with vasopressors. ${ }^{4)}$

Macrophages are the most commonly occurring leukocytes that are closely related to the development of sepsis. ${ }^{5)}$ The M1 differentiation of macrophages is enhanced in the early stage of sepsis, probably due to stimulation by cytokines, including interferon- $\gamma$ and granulocyte macrophage colony stimulating factor, and exogenous bacterial toxins, such as lipopolysaccharide (LPS). M1 macrophages are "classically activated" macrophages that are characterized by an increased ability to secrete pro-inflammatory cytokines, including interleukin (IL)-1 $\beta$, tumor necrosis factor (TNF)- $\alpha$, high mobility group 1 (HMGB1) protein, IL-12, and IL-18. ${ }^{6}$ ) These cytokines improve the ability of the immune system to eliminate microorganisms, however, they also cause tissue damage. Moreover, the overproduction of cytokines in the early hyperinflammatory stage induces immunosuppression in the later stages of sepsis, although the mechanism underlying this phenomenon is yet to be fully understood. A moderate inhibition of the immune response is therefore recommended during the hyperinflammatory phase.

Recent studies have demonstrated that the rate of glycolysis in macrophages is enhanced during the stage of immune activation, however, it is attenuated during the stage of immune tolerance, which suggests a correlation between glycolysis and the immune function. ${ }^{7)}$ The activated immune cells, including macrophages, dendritic cells, and $\mathrm{T}$ cells, also have the ability to switch from oxidative phosphorylation to aerobic glycolysis even when oxygen is abundantly available. LPS has been reported to promote the expression of genes related to glycolysis via activation of the hypoxia inducible factor (HIF) and mammalian target of rapamycin (mTOR) signaling pathways. ${ }^{8}$ It has been demonstrated that the inhibition of glycolysis impairs the LPS-induced production of IL- $1 \beta$ by macrophages. This indicates that glycolysis mediates the LPS-induced activation of the immune system. ${ }^{9)}$ Pyruvate kinase 2 (PKM2) plays an important role in glycolysis by acting as a protein kinase and transcriptional coactivator. PKM2 catalyzes the conversion of phosphoenolpyruvic acid (PEP) and ADP to pyruvate and ATP, respectively, in the last step of the glycolytic pathway. ${ }^{10)}$ 
PKM2 can also function as a transcriptional coactivator of HIF-1 $\alpha$ for stimulating the expression of the glycolytic enzymes and proinflammatory cytokines. ${ }^{11)}$

Lycium barbarum polysaccharide (LBP) is the main bioactive component of Chinese wolfberry that has several properties, including anti-inflammatory, anti-oxidative, anti-aging, and hypolipidemic activities. ${ }^{12}$ The anti-inflammatory effects of LBP have been reported in various diseases. ${ }^{13-15)}$ For instance, LBP protects the kidneys from inflammatory injury in septic rats by regulating the Keap1-nuclear factor-E2-related factor 2 (Nrf2)/antioxidant response element (ARE) signaling pathway. ${ }^{13)}$ Additionally, LBP attenuates the LPS-induced acute respiratory distress syndrome by inhibiting inflammation, apoptosis, and oxidative stress in pulmonary endothelial cells. ${ }^{14)}$ LBP also alleviates the inflammation induced by IL- $1 \beta$ in chondrogenic ATDC5 cells by modulating miR-124. ${ }^{15)}$

LBP has been reported to suppress the expression of genes related to glycolysis and gluconeogenesis, in a model of insulin resistance induced by the long-term administration of a high-fat diet. ${ }^{12)}$ We therefore investigated whether LBP suppresses the LPS-induced inflammatory response in macrophages by suppressing glycolysis. This study confirmed that LBP inhibited the LPS-induced glycolysis in macrophages by reducing the levels of the PKM2 protein. The reduction in the levels of PKM2 by LBP was associated with the ubiquitination and degradation of PKM2. The results of this study elucidated a novel anti-inflammatory effect of LBP on macrophages.

\section{MATERIALS AND METHODS}

Cell Culture and Treatment Murine RAW264.7 macrophages were obtained from the Cell Bank of the Chinese Academy of Sciences (Shanghai, China). The RAW264.7 macrophages were initially cultured in LPS-free Dulbecco's modified Eagle medium (DMEM, Gibco Company, Grand Island, NY, U.S.A.), supplemented with $10 \%$ fetal bovine serum (Gibco), $100 \mathrm{U} / \mathrm{mL}$ streptomycin (Invitrogen, Carlsbad, CA, U.S.A.), and $100 \mathrm{U} / \mathrm{mL}$ penicillin (Invitrogen), in an incubator with $5 \% \mathrm{CO}_{2}$ and saturated humidity.

The RAW264.7 macrophages were treated with 10, 50, or $200 \mu \mathrm{g} / \mathrm{mL}$ LBP (No. 107-43-7; Guidechem Chemical Com- pany, Shaanxi, China, https://china.guidechem.com/trade/ pdetail1649419.html), alone or in combination with $100 \mathrm{ng} / \mathrm{mL}$ LPS (Sigma-Aldrich, St. Louis, MO, U.S.A.), for $24 \mathrm{~h}$. Following treatment, the cells were subjected to several analyses that are described hereafter.

3-(4,5-Dimethylthiazol-2-yl)-2,5-diphenyltetrazolium Bromide (MTT) Assay The cell viability was evaluated by the MTT assay. The RAW264.7 macrophages were seeded into a 96-well plate at a density of $1 \times 10^{4}$ cells/well, and three parallel wells were set up for each group. The cells were incubated with $20 \mu \mathrm{L} \mathrm{MTT} \mathrm{solution} \mathrm{(Sigma-Aldrich)} \mathrm{for} 4 \mathrm{~h}$ at $37^{\circ} \mathrm{C}$. The MTT solution was subsequently removed and dimethyl sulfoxide (DMSO, $150 \mu \mathrm{L}$, Sigma-Aldrich) was added to each well. The values of optical density (OD) were determined for each well using an Infinite F500 microplate reader (TECAN, Männedorf, Switzerland) at a wavelength of $490 \mathrm{~nm}$.

Measurement of Glucose Concentration in the Culture Medium The culture medium was collected for measuring the concentration of extracellular glucose. The glucose concentration was determined using the conventional glucose oxidase/peroxidase method that has been previously described. ${ }^{16)}$ This method is based on the conversion of glucose and $\mathrm{O}_{2}$ into gluconic acid and $\mathrm{H}_{2} \mathrm{O}_{2}$ by glucose oxidase. The $\mathrm{H}_{2} \mathrm{O}_{2}$ thus produced further reacts with 1,5-dimethyl-2-phenyl-4aminopyrazoline and phenols, and generates colored compounds with a maximum absorption wavelength of $570 \mathrm{~nm}$.

Measurement of Lactic Acid Concentration The concentration of lactic acid in the culture medium was determined using a lactic acid assay kit (Item number: K607-100, Biovision; Wuhan, China), according to the manufacturer's instructions. This kit consists of a highly sensitive probe that emits fluorescence $(E x / E m=535 / 587 \mathrm{~nm})$ after reacting with lactic acid. The emitted fluorescence was detected with an Infinite F500 microplate reader (TECAN).

Enzyme-Linked Immunosorbent Assay (ELISA) The RAW264.7 macrophages were cultured in 24-well plates at a density of $10^{6}$ cells/well and treated with the aforementioned substances. The culture supernatants were collected and centrifuged in order to remove the particles for subsequent analyses. The cell supernatants were collected and stored at $-20^{\circ} \mathrm{C}$ until further analysis. The quantities of IL- $1 \beta$, TNF- $\alpha$, and

Table 1. The Information of Primer in PCR Assay

\begin{tabular}{|c|c|c|c|c|c|c|}
\hline Genes & Direction & Sequence $\left(5^{\prime} \rightarrow 3^{\prime}\right)$ & Length & $T_{\mathrm{m}}$ & Location & Amplification size \\
\hline PKM2 & Forward & ATGTCGAAGCCCCATAGTGAA & 21 & 60.9 & $1-21$ & 118 \\
\hline PKM2 & Reverse & TGGGTGGTGAATCAATGTCCA & 21 & 61.4 & $118-98$ & \\
\hline IL-4 & Forward & CCAACTGCTTCCСССТСТG & 19 & 62 & $15-33$ & 150 \\
\hline IL-4 & Reverse & TCTGTTACGGTCAACTCGGTG & 21 & 61.7 & $164-144$ & \\
\hline IL-10 & Forward & GACTTTAAGGGTTACCTGGGTTG & 23 & 60.5 & $217-239$ & 112 \\
\hline IL-10 & Reverse & TCACATGCGCCTTGATGTCTG & 21 & 63 & $328-308$ & \\
\hline NEDD4L & Forward & GACATGGAGCATGGATGGGAA & 21 & 61.9 & $481-501$ & 122 \\
\hline NEDD4L & Reverse & GTTCGGCCTAAATTGTCCACT & 21 & 60.3 & $602-582$ & \\
\hline NEDD4 & Forward & TCAGGACAACCTAACAGATGCT & 22 & 60.8 & $672-693$ & 223 \\
\hline NEDD4 & Reverse & TTCTGCAAGATGAGTTGGAACAT & 23 & 60.2 & $897-875$ & \\
\hline Gnb2 & Forward & TGATGCCTCTATCAAGCTGTGG & 22 & 61.8 & $612-633$ & 76 \\
\hline $\mathrm{Gnb} 2$ & Reverse & GATGTCGGATTCATGGCCGAT & 21 & 62.3 & $687-667$ & \\
\hline Smurf1 & Forward & AGATCCGTCTGACAGTGTTATGT & 23 & 60.8 & $41-63$ & 92 \\
\hline Smurf1 & Reverse & CCCATCCACGACAATCTTTGC & 21 & 61.6 & $132-112$ & \\
\hline GAPDH & Forward & GGAGCGAGATCCCTCCAAAAT & 21 & 61.6 & $108-128$ & 197 \\
\hline GAPDH & Reverse & GGCTGTTGTCATACTTCTCATGG & 23 & 60.9 & $304-282$ & \\
\hline
\end{tabular}




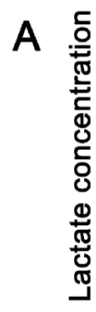
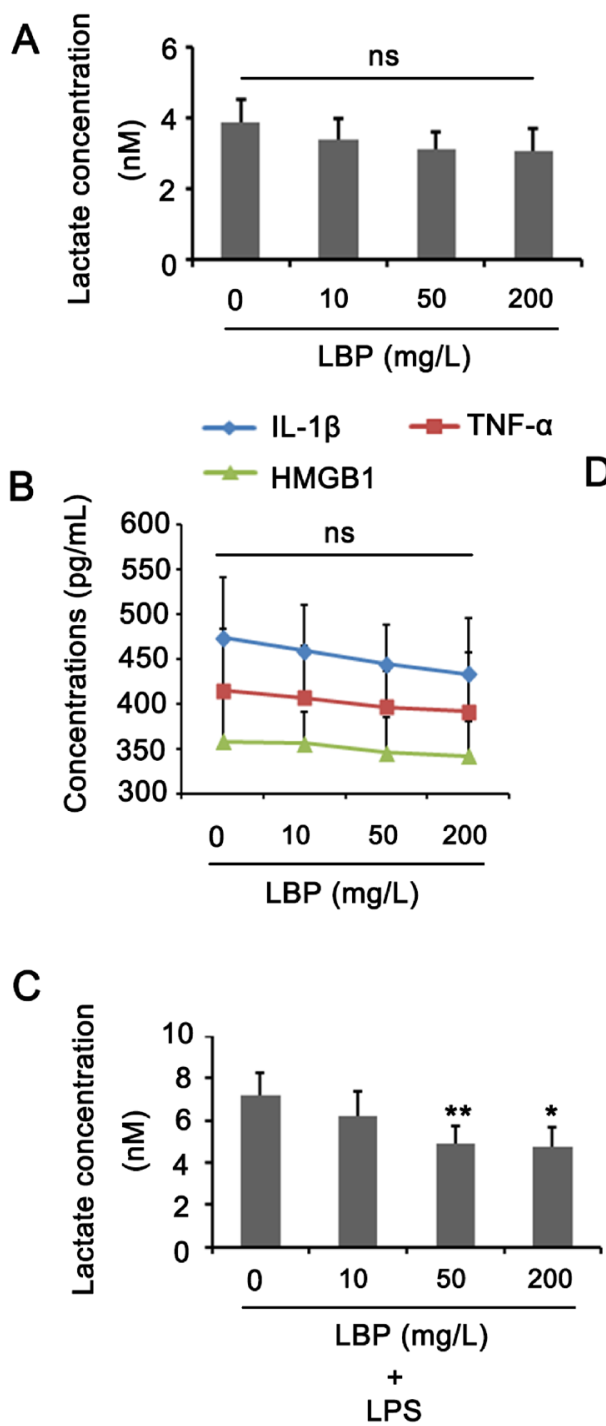
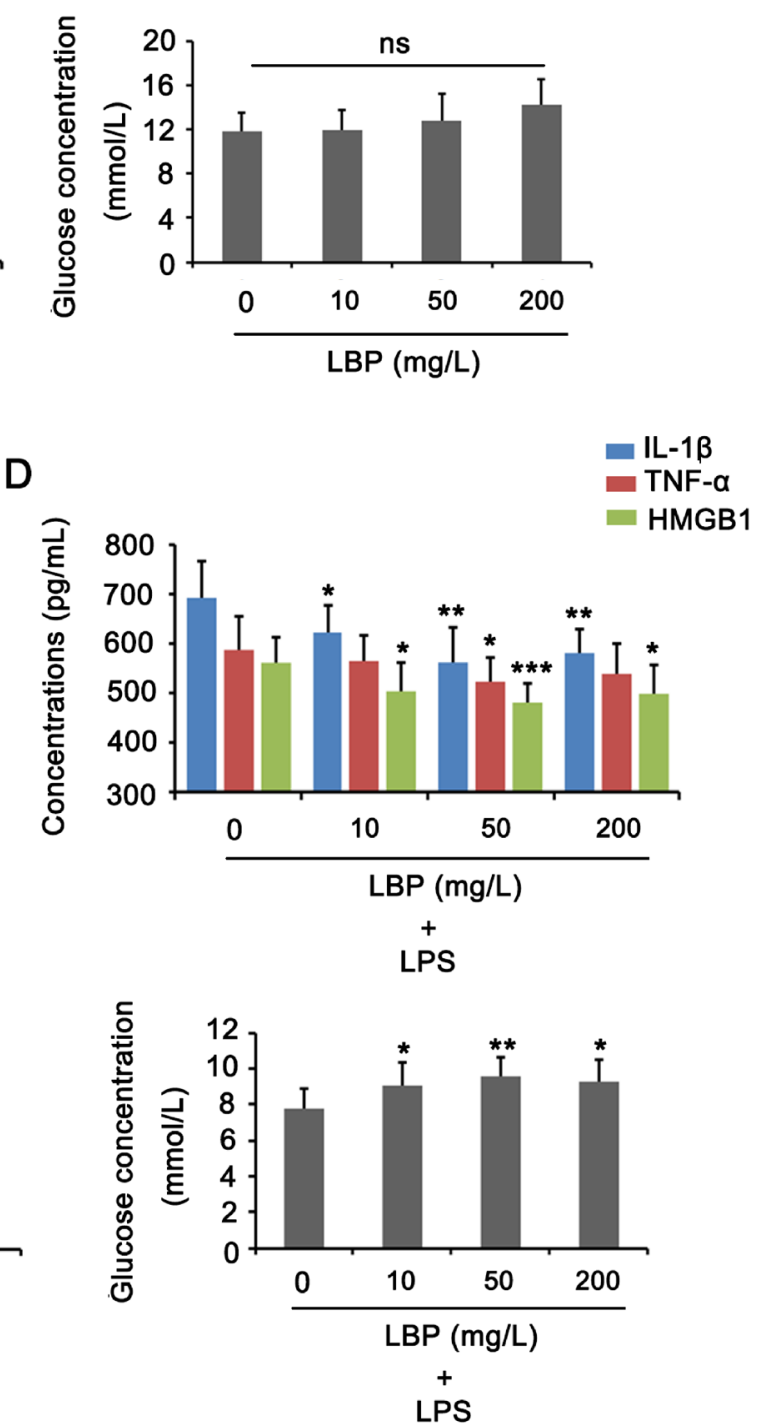

Fig. 1. Effects of LBP on Glycolysis and the Production of Inflammatory Factors in Macrophage in the Presence or Absence of LPS

Macrophages were treated with 10, 50, and $200 \mu \mathrm{g} / \mathrm{mL}$ LBP for $24 \mathrm{~h}$. The concentrations of lactate and glucose in the culture medium of macrophages were assessed after treatment with LBP (A). The concentrations of IL-1 $\beta$, TNF- $\alpha$ and HMGB1 in the culture medium were measured using Elisa assay (B). Macrophages were treated with 10,50 , and $200 \mu \mathrm{g} / \mathrm{mL}$ LBP in combination with $100 \mathrm{ng} / \mathrm{mL}$ LPS for $24 \mathrm{~h}$. The culture medium was collected for the measurements of lactate and glucose concentrations (C) as well as IL-1 $\beta$, TNF- $\alpha$ and HMGB1 concentrations (D). ns: no significance. ${ }^{*} p<0.05, * * p<0.01$, and $* * * p<0.01 v s$. control. (Color figure can be accessed in the online version.)

HMGB1 in the culture medium were assessed using a commercially available ELISA kit, according to the manufacturer's instructions (Sigma-Aldrich). The value of OD of each well was measured at a wavelength of $450 \mathrm{~nm}$. The experiment was performed in triplicate, and the mean value was calculated. The IL-1 $\beta$ ELISA assay kit does not distinguish between the inactive $31-\mathrm{kDa}$ precursor (pro-IL-1 $\beta$ ) and the bioactive $17 \mathrm{kDa}$ mature form, as is the case for all commercially available ELISA kits. The standard curves were used for measuring the concentrations of IL- $1 \beta$ in the samples $(\mathrm{pg} / \mathrm{mL})$.

RT-Quantitative PCR The total RNA was extracted using the single-step TRIzol method (Invitrogen) and reversetranscribed to cDNA using the reverse transcriptase of avian myeloblastosis virus. The primers for PCR were designed and synthesized by Invitrogen, and are enlisted in Table 1. Glyceraldehyde-3-phosphate dehydrogenase (GAPDH) was used as the internal reference. The following amplification conditions were used for PCR: pre-denaturation at $94^{\circ} \mathrm{C}$ for $5 \mathrm{~min}$, denaturation at $94^{\circ} \mathrm{C}$ for $40 \mathrm{~s}$, annealing at $60^{\circ} \mathrm{C}$ for $40 \mathrm{~s}$, and extension of the DNA strands at $72^{\circ} \mathrm{C}$ for $1 \mathrm{~min}$. This protocol was run for 40 cycles, followed by extension at $72^{\circ} \mathrm{C}$ for $10 \mathrm{~min}$. The $2^{-\Delta \Delta \mathrm{Ct}}$ method was used for calculating the relative expression of the target genes using to the following formula: $\Delta \Delta \mathrm{Ct}=(\mathrm{Ct}[$ target gene $]-\mathrm{Ct}[$ reference gene] $)$ experimental group - LRB - Ct[target gene] $-\mathrm{Ct}[$ reference gene])control group.

Analysis by Western Blotting The proteins that were extracted from the cells were mixed with the loading buffer and boiled for $10 \mathrm{~min}$ at $95^{\circ} \mathrm{C}$. The protein samples were loaded onto a $10 \%$ polyacrylamide gel and separated by electrophoresis (Boster Biological Technology, Wuhan, China). The proteins were transferred onto a polyvinylidene fluoride membrane and the membrane was blocked with $5 \%$ bovine serum albumin for $1 \mathrm{~h}$ at room temperature. The membrane was incubated overnight at $4^{\circ} \mathrm{C}$ with the primary antibodies against PKM2, phosphorylated (p)-IRAK4, IRAK4, p-IRAK1, IRAK4, Nedd4L, Nedd4, Gnb2, Smurf1, HIF-1 $\alpha$, and GAPDH (1:1000, Abcam Inc., Cambridge, MA, U.S.A.). The mem- 
brane was subsequently washed with Tris-buffered saline in Tween-20, and subsequently treated with the secondary antibodies (Jackson ImmunoResearch Laboratories, West Grove, PA, U.S.A.) for $1 \mathrm{~h}$. The images of the gels were captured using a Bio-Rad Gel Doc EZ Imager (Bio-Rad, Hercules, CA, U.S.A.). The target protein bands were analyzed in terms of the gray values using the Image J software.

Gene Knockdown and Overexpression Three sets of small interfering RNAs (siRNAs) against PKM2 were designed by GenePharma (Shanghai, China) and the efficiency of PKM2 knockdown was initially determined. The siRNA with the highest PKM2 knockdown efficiency was used for further studies. For inducing the ectopic expression of PKM2 in RAW264.7 macrophages, the cells were transfected with the pEGFP-C1-PKM2 vector (Genephama Biotech) using Lipofectamine 2000 (Thermo Fisher Scientific, Waltham, MA, U.S.A.), according to the manufacturer's instructions.

Flow Cytometry CD86 (BioLegend, San Diego, CA, U.S.A.) is a surface marker of M1 macrophages, while CD206 (BioLegend) is a surface marker of the M2 macrophages. The cells were harvested by centrifugation, and the supernatant was discarded. The cells were washed with pre-cooled phosphate buffered saline (PBS) buffer at $4{ }^{\circ} \mathrm{C}$, and subsequently centrifuged twice at $1000 \mathrm{rpm}$, for $5 \mathrm{~min}$ each. The cell density was adjusted to $1 \times 10^{6}$ cells $/ \mathrm{mL}$. The cell suspension was subsequently washed twice with pre-cooled PBS and centrifuged. The cells were suspended in $300 \mu \mathrm{L}$ binding buffer and incubated with anti-IgG1 labeled with phycoerythrin (PE), PE-cyanine 5 (Cy5), or fluorescein isothiocyanate (FITC), which were used as the negative controls, or with PE-Cy5conjugated anti-CD86 or FITC-conjugated anti-CD206. After $30 \mathrm{~min}$ of incubation, approximate 30000 cells were selected from each sample and suspended in $300 \mu \mathrm{L}$ of PBS. The cells were subsequently analyzed by flow cytometry using a BD LSRFortessa flow cytometer within $24 \mathrm{~h}$. The FlowJo software was used for data analysis.

Co-immunoprecipitation (Co-IP) Assay The whole cell extracts were prepared using IP lysis buffer containing $10 \mathrm{mM}$ Tris- $\mathrm{HCl}, 5 \mathrm{mM}$ ethylenediaminetetraacetic acid (EDTA), $50 \mathrm{mM} \mathrm{NaCl}, 50 \mathrm{mM} \mathrm{NaF}$, and $1 \%$ Triton $\times 100$ supplemented with the complete protease inhibitor cocktail and PhosStop tablets (Roche, Basel, Switzerland). The lysates $(0.5-1.5 \mathrm{mg})$ were incubated with $2 \mu \mathrm{g}$ of PKM2 antibody and Protein GSepharose (GE Healthcare, Chicago, IL, U.S.A.). The PKM2protein complexes were subjected to Western blotting for determining the enrichment of HIF- $1 \alpha$ and ubiquitin in the protein complex.

Statistical Analyses The experiments were repeated thrice, and the mean values were calculated. All the data were analyzed with SPSS software, version 21.0 (SPSS Inc., Chicago, IL, U.S.A.). The quantitative data are expressed as the mean \pm standard deviation. The normally distributed quantitative data were compared using $t$-tests, while one-way ANOVA was performed for comparing the multiple groups. Statistical significance was defined at $p<0.05$.

\section{RESULTS}

LBP Had Moderate Effects on Glycolysis and the Production of Inflammatory Factors in Macrophages The effect of LBP on glycolysis has not been previously investi- gated in macrophages. In order to determine the effects of LBP on macrophages, we determined the concentrations of lactate and glucose in the culture medium of macrophages following treatment with LBP. We found that the concentrations of lactate and glucose in the culture medium were not significantly altered following treatment with 10, 50, and $200 \mu \mathrm{g} / \mathrm{mL}$ of LBP (Fig. 1A). We observed that LBP significantly reduced cell viability at concentrations of $400 \mu \mathrm{g} / \mathrm{mL}$, but not at lower concentrations (data not shown). We therefore performed the subsequent experiments using LBP at concentrations $<200 \mu \mathrm{g} / \mathrm{mL}$. The concentrations of IL- $1 \beta$, TNF- $\alpha$, and HMGB1 in the culture medium remained unaltered following treatment with LBP (Fig. 1B).

LBP Suppressed Glycolysis and the Production of Inflammatory Factors in the Macrophages in the Presence of LPS Co-treatment with LPS and LBP decreased the concentrations of lactate in the culture medium, compared to those after treatment with LPS alone $(p<0.05$ or $p<0.01$, Fig. 1C). The inhibition induced by $50 \mu \mathrm{g} / \mathrm{mL}$ LBP was more significant than that of $200 \mu \mathrm{g} / \mathrm{mL}$ LBP. Treatment with LPS and LBP at all the tested concentrations increased the concentrations of glucose in the culture medium, compared to those induced by treatment with LPS alone $(p<0.05$ or $p<0.01$, Fig. 1C). The results of ELISA indicated that LBP reduced the concentrations of IL- $1 \beta$, TNF- $\alpha$, and HMGB1 in the culture medium in the presence of LPS. The inhibitory potential of $50 \mu \mathrm{g} / \mathrm{mL}$ of LBP was significantly higher than that of LBP at all the other concentrations tested herein. We therefore performed further experiments with $50 \mu \mathrm{g} / \mathrm{mL} \mathrm{LBP}$.

The Effects of LBP against LPS-Induced Glycolysis and Inflammation Are Mediated via PKM2 In this study we performed PCR and Western blotting for determining the mRNA and protein levels of PKM2, respectively, in the macrophages following treatment with LPS alone or in combination with LBP. LPS increased the mRNA levels of PKM2 in the macrophages in the presence or absence of LBP $(p<0.05$, Fig. 2A). The protein levels of PKM2 were markedly increased following treatment with LPS $(p<0.001$, Fig. 2B), whereas treatment with LBP suppressed the increase in the protein levels of PKM2 that had been induced by LPS $(p<0.001 v s$. LPS group). In order to determine whether the effects of LBP on LPS-induced glycolysis and inflammation are mediated via PKM2, we transfected the macrophages with PKM2-siRNA or an overexpression vector prior to treatment with LPS and/or LBP. Transfection with the PKM2 overexpression vector dramatically increased the mRNA levels of PKM2 in the macrophages following treatment with LPS and LBP $(p<0.01$ vs. LPS group, Fig. 2C). Transfection with PKM2-siRNA in turn reduced the mRNA levels of PKM2 in the macrophages following exposure to LPS ( $p<0.001 v s$. LPS group, Fig. 2C). LBP restrained the LPS-induced increase in the protein levels of PKM2, however, transfection with the PKM2 overexpression vector before these treatments increased the protein levels of PKM2 $(p<0.001 v s$. control group, Fig. 2D). Transfection with PKM2-siRNA also abolished the LPS-induced increase in the protein levels of PKM2 ( $p<0.001 v s$. LPS group).

As depicted in Fig. 3A, LPS increased the concentration of lactate in the culture medium ( $p<0.001 v s$. control group). The increase in the concentration of lactate was inhibited by LBP ( $p<0.01 v s$. LPS group). However, the inhibitory effect of LBP was antagonized by the forced expression of 

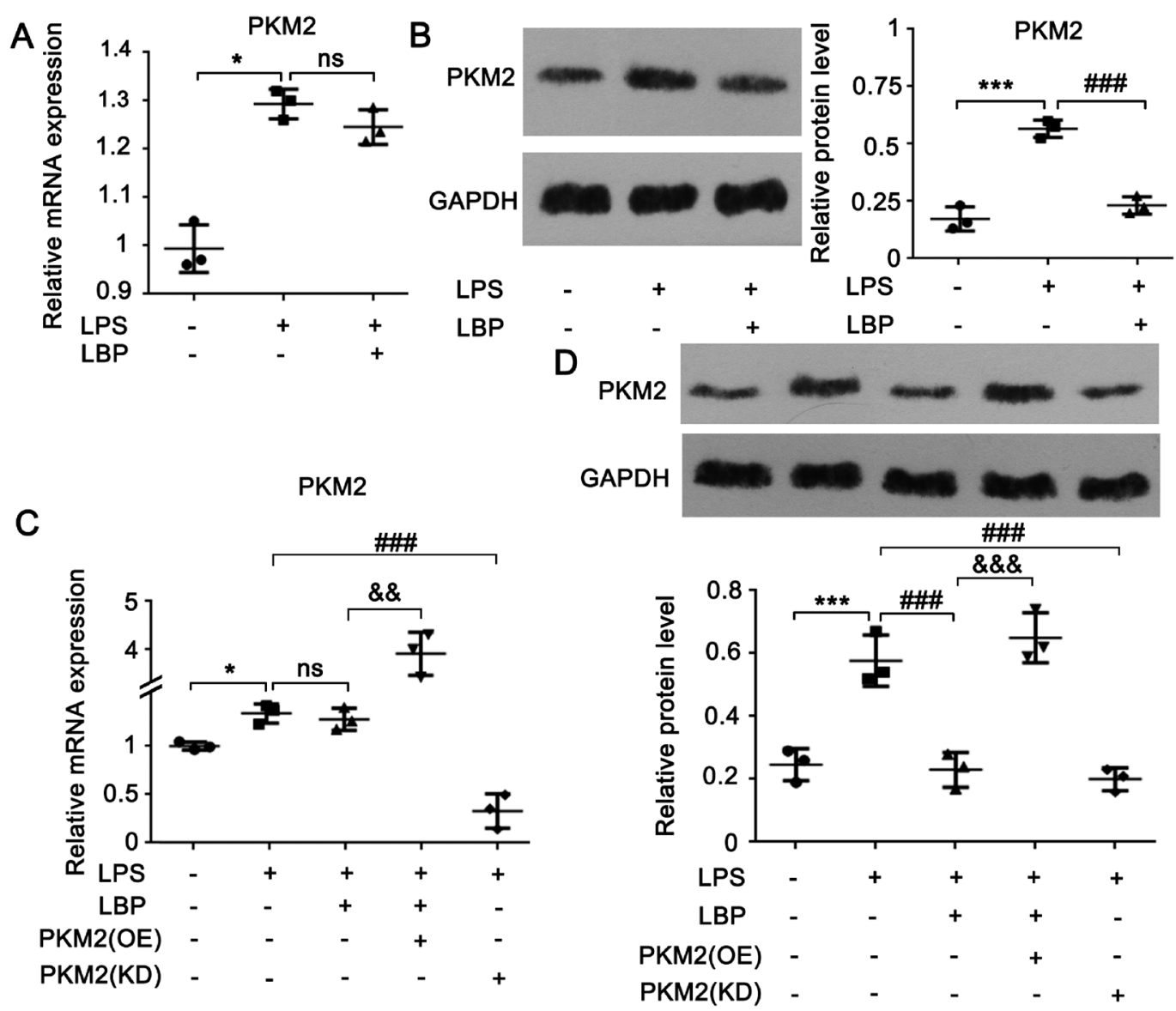

Fig. 2. Knockdown or Overexpression of PKM2 in Macrophages before Treatment with LPS and/or LBP

This study performed PCR (A) and Western blot (B) assays to assess PKM2 mRNA and protein levels, respectively, in macrophages after treatments with LPS alone or in combination with LBP. PKM2-siRNA or over-expression vector was transfected into macrophages, before treatment with LPS and/or LBP. PCR (C) and Western blot (D) assays was performed to assess PKM2 mRNA and protein levels. ${ }^{*} p<0.05$, and ${ }^{*} * * p<0.01 v s$. control; ${ }^{\# \# \#} p<0.01 v s$. LPS group. \&\& $p<0.01$, and ${ }^{\& \& \&} p<0.01 v s$. LPS + LBP group.

PKM2 by transfection with the PKM2 overexpression vector ( $p<0.01$ vs. LPS + LBP group). The silencing of PKM2 also suppressed the LPS-induced increase in the concentration of lactate, similar to the inhibitory effect of LBP $(p<0.01$ $v s$. LPS group). LPS decreased the concentration of glucose in the culture medium ( $p<0.01 v s$. control group, Fig. 3B), while LBP antagonized the LPS-induced reduction in the concentration of glucose ( $p<0.01 v s$. LPS group). The overexpression of PKM2 abolished the inhibitory effect of LBP on the LPS-induced reduction in the concentration of glucose $(p<0.01 v s$. LPS + LBP group). PKM2 knockdown reversed the LPS-induced reduction in the concentration of glucose ( $p<0.001 v s$. LPS group). The results of ELISA demonstrated that the concentrations of IL- $1 \beta$, TNF- $\alpha$, and HMGB1 in the culture medium were elevated following treatment with LPS $(p<0.001, p<0.01$, and $p<0.001$, respectively, Fig. 3C). LBP antagonized the increase in the concentrations of IL- $1 \beta$, TNF- $\alpha$, and HMGB1 that was stimulated by LPS $(p<0.01$, $p<0.05$, and $p<0.001$, respectively, vs. LPS group), however, the overexpression of PKM2 abrogated this effect of LBP $(p<0.01, p<0.01$ and $p<0.001$, respectively, $v s$. LPS + LBP group). PKM2 knockdown also suppressed the increase in the concentrations of IL- $1 \beta$, TNF- $\alpha$, and HMGB1 that were induced by treatment with LPS $(p<0.001, p<0.05$, and $p<0.01$, respectively, $v s$. LPS group).
LBP Disrupted the Interaction between PKM2 and HIF$1 \alpha$, and Suppressed the Differentiation of M1 Macrophages We performed Western blotting and Co-IP assays for determining the interaction between PKM2 and HIF- $1 \alpha$ in the macrophages following treatment with LPS and LBP. The results of Western blotting revealed that LPS increased the levels of HIF- $1 \alpha$ protein (input) in the macrophages $(p<0.001$, Fig. $4 \mathrm{~A})$. The LPS-induced increase in the levels of HIF-1 $\alpha$ protein were unaffected by treatment with LBP. Treatment with LPS increased the enrichment of HIF-1 $\alpha$ in the PKM2 protein (IB, $p<0.001$ ), as revealed by the results of Co-IP. However, LBP suppressed the increase in the enrichment of HIF- $1 \alpha$ in the PKM2 protein ( $p<0.05$ vs. LPS group).

The M1/M2 differentiation of macrophages was assessed by flow cytometry for determining the cell surface markers of M1 (CD86) and M2 (CD206) macrophages. Treatment with LPS increased the percentage of macrophages with M1 phenotype $(p<0.001$, Fig. 4B), but decreased the percentage of macrophages with M2 phenotype $(p<0.01)$. LBP suppressed the effects of LPS on the M1/M2 differentiation of macrophages ( $p<0.01$ vs. LPS group). The expression of IL-4 and IL-10 mRNA in the macrophages with M2 phenotype was additionally investigated by PCR. The expression levels of IL-4 and IL-10 mRNA reduced following stimulation with LPS $(p<0.001$, Fig. $4 C)$. LBP reversed the LPS-induced reduction in the expression of IL-4 and IL-10 mRNA ( $p<0.001$ vs. LPS group). 


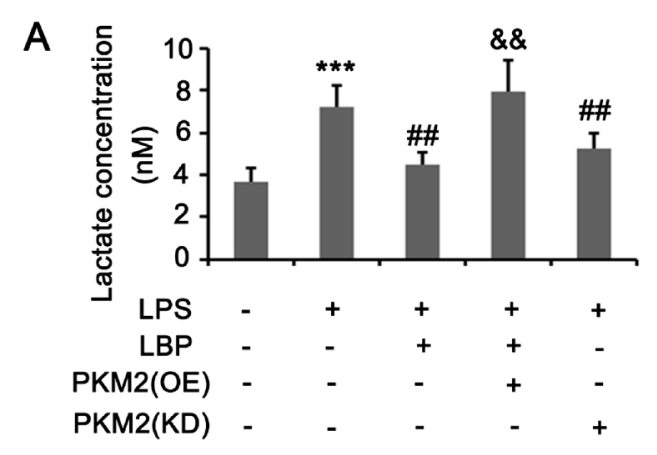

$\mathrm{C}$

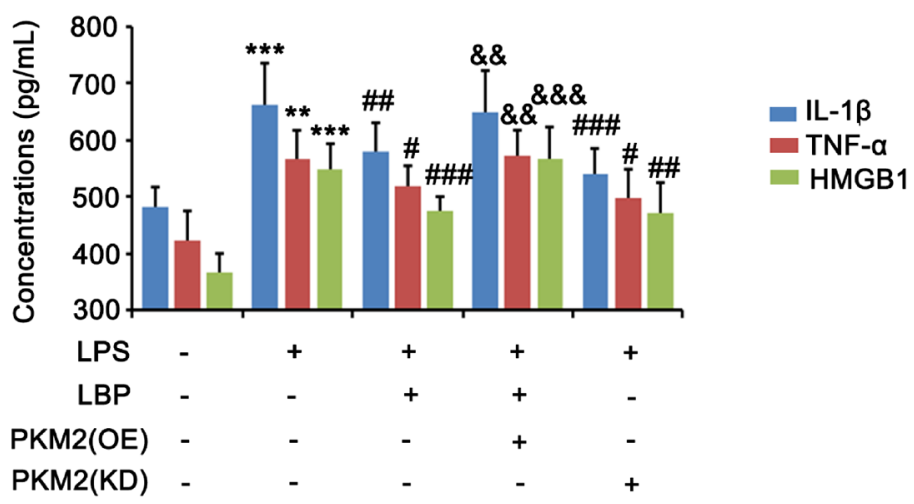

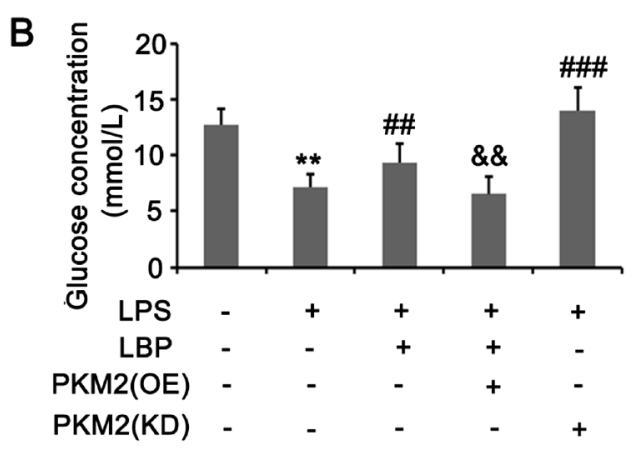

Fig. 3. PKM2 Was Involved in the Effects of LBP against LPS-Induced Glycolysis and Inflammation

This study performed PCR (A) and Western blot (B) assays to assess PKM2 mRNA and protein levels, respectively, in macrophages after treatments with LPS alone or in combination with LBP. PKM2-siRNA or over-expression vector was transfected into macrophages, before treatment with LPS and/or LBP. PCR (C) and Western blot (D) assays was performed to assess PKM2 mRNA and protein levels. The culture medium was collected for the measurements of lactate (E) and glucose concentrations (F) as well as IL- $1 \beta$, TNF- $\alpha$ and HMGB1 concentrations (G). ns: no significance. ${ }^{*} p<0.05, * * p<0.01$, and $* * * p<0.01 v s$. control; ${ }^{\#} p<0.05$, \# $p<0.01$, and ${ }^{\# \# \# ~} p<0.01$ vs. LPS group. (Color figure can be accessed in the online version.)

LPS Increased the Expression of PKM2 Protein by Suppressing LBP-Induced Disruption of PKM2 Ubiquitination It was observed that LBP suppressed the LPS-induced increase in the expression of the PKM2 protein but not that of the PKM2 mRNA, which suggested that the modulatory effect of LBP on the PKM2 protein is mediated by a posttranscriptional mechanism. Bioinformatics analysis (http:// ubibrowser.ncpsb.org/ubibrowser/) revealed that the PKM2 protein is ubiquitinated by a series of ubiquitin ligases, including Nedd4L, Nedd4, Gnb2, and Smurf1. Data from the GSE76562 dataset (https://www.ncbi.nlm.nih.gov/geo/ query/acc.cgi?acc $=$ GSE76562 $)^{17)}$ revealed that LPS downregulates the expression of Nedd4L, Nedd4, and Gnb2, but has a moderate effect on the expression of Smurf1. Based on this information, we investigated whether LBP disrupts the effect of LPS on the expression of the ubiquitin ligases. The results of PCR revealed that LPS downregulated the expression of Nedd4L $(p<0.001)$, Nedd4 $(p<0.001)$, and Gnb2 $(p<0.01)$ (Fig. 5A). LBP reversed the LPS-induced increase in the expression of Nedd4L $(p<0.001 v s$. LPS group) and Nedd4 ( $p<0.05$ vs. LPS group). The protein levels of Nedd4L $(p<0.001)$, Nedd4 $(p<0.001)$, Gnb2 $(p<0.01)$, and Smurf1 $(p<0.05)$ decreased following treatment with LPS (Fig. 5B). LBP reversed the increase in the protein levels of Nedd4L ( $p<0.001 v s$. LPS group), Nedd4 ( $p<0.05 v s$. LPS group), and Gnb2 ( $p<0.05$ vs. LPS group) that were induced by LPS. However, treatment with LBP alone, at least at the concentration of $50 \mu \mathrm{g} / \mathrm{mL}$, had no effect on the expression level of NEDD4, NEDD4L, and Gnb2 (data not shown). Analysis of the results of Co-IP indicated that LPS enhanced the ubiqui- tination of PKM2; however, this effect of LPS was impaired following treatment with LBP (Fig. 5C).

LBP Had No Effect on the Activation of Toll-Like Receptor (TLR)4 by LPS To determine whether LBP interacts with LPS and inhibits the effect of LPS on TLR4 activation, this study investigated the downstream signal of TLR4 after treatment with LPS alone or in combination with LBP. Upon LPS stimulation, the intracellular TIR region of TLR4 binds to the carboxyl terminus of MAL and MyD88. MyD88 further recruits IRAK4 (IL-1 receptor-associated kinase-4), IRAK1, and IRAK2 and results in their phosphorylation. ${ }^{18)}$ IRAKs interact with TAK1, whereby influencing IKK $\alpha$ / inhibitor of kappaB (I $\kappa \mathrm{B}) /$ nuclear factor-kappaB (NF- $\kappa \mathrm{B})$ signal. Therefore, the phosphorylation status of IRAKs, such as IRAK4 and IRAK1, has been used to evaluate the activation of TLR4 signal by LPS. This study found that LPS induced the phosphorylation of IRAK4 and IRAK1 $(p<0.001$, Fig. $6)$. LBP failed to reverse the phosphorylation of IRAK4 and IRAK1 induced by LPS. The protein levels of IRAK4 and IRAK1 were neither influenced by LPS nor LPS plus LBP. This result suggested that LBP had no effect on the stimulation of TLR4 by LPS.

\section{DISCUSSION}

The anti-inflammatory effect of LBP has been reported in various studies. ${ }^{13-15)}$ The results of this study demonstrated that the treatment of macrophages with LBP alone had no effect on the secretion of the inflammatory factors, including IL- $1 \beta$, TNF- $\alpha$, and HMGB1. However, LBP suppressed the 
A1

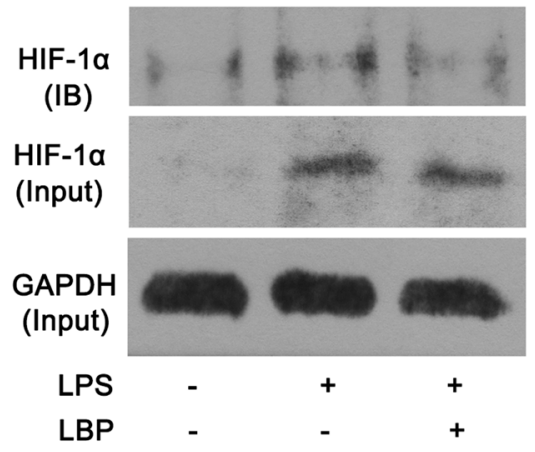

A2



B1
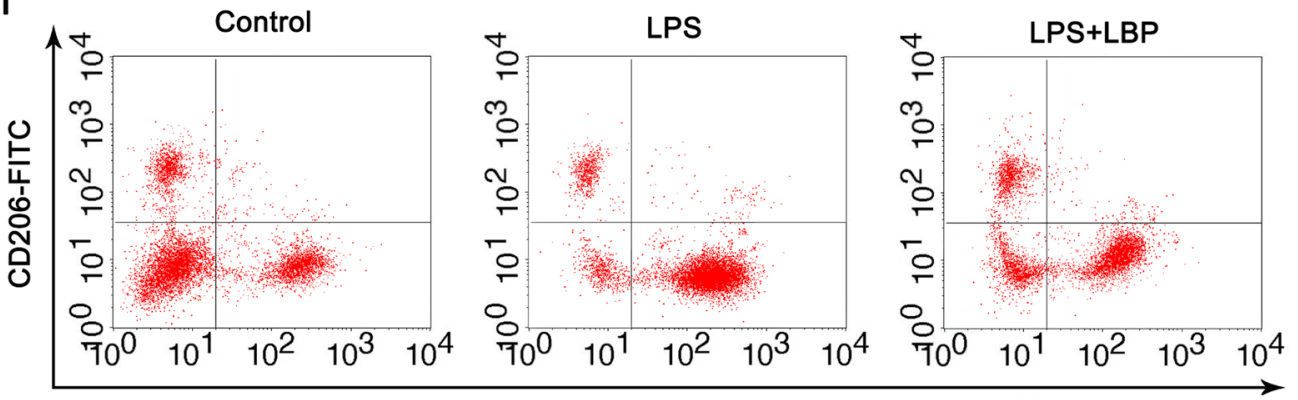

CD86-PE-Cy5
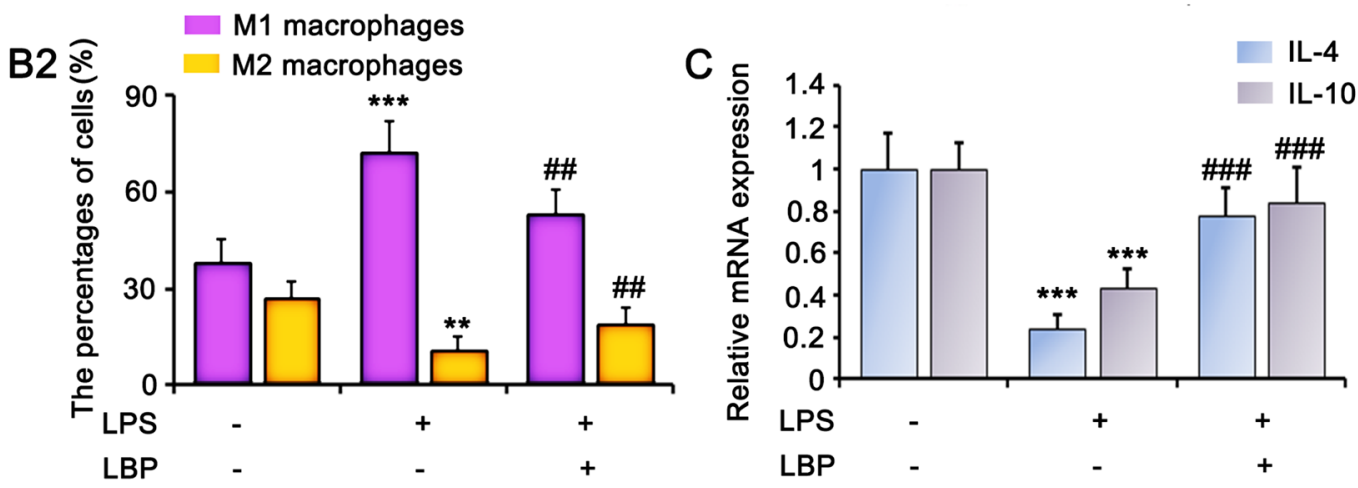

Fig. 4. LBP Disrupted the Interaction between PKM2 and HIF-1 $\alpha$ and Suppressed M1 Differentiation of Macrophage

This study performed Western blot and Co-IP assays to determine the interaction between PKM2 and HIF-1 $\alpha$ in macrophages after treatment with LPS alone or in combination with LBP (A). The M1/M2 differentiation of macrophages was assessed through flow cytometry assay for the testing cell surface markers of M1 phenotype (CD86) and M2 phenotype (CD206) (B). PCR assay was performed to test IL-4 and IL-10 mRNA expression in macrophages (C). ** $p<0.01$, and $* * * p<0.01$ vs. control; ${ }^{\#} p<0.05,{ }^{\#} p<0.01$, and ${ }^{\# \#} p<0.01 v s$. LPS group. (Color figure can be accessed in the online version.)

secretion of IL-1 $\beta$, TNF- $\alpha$, and HMGB1 in the presence of LPS. These data suggested that the anti-inflammatory effects of LBP depend on the cellular environment.

Accumulating evidence suggests the important role of glycolysis in the activation and polarization of macrophages. Zhong et al. demonstrated that 2-deoxyglucose (2-DG), an inhibitor of glycolysis, markedly attenuates the production of proinflammatory factors and lung tissue injury in a murine model of LPS-induced acute lung injury. ${ }^{19)}$ Using in vitro and in vivo experiments, Tannahill et al. also reported that 2-DG suppresses the LPS-induced production of IL- $1 \beta$ in macrophages.9) These observations agree with the finding that the rate of glycolysis in macrophages with the M1 phenotype is higher than that of the macrophages with the M2 phenotype. ${ }^{20-22)}$ Glycolysis is essential for maintaining the M1 macrophages as the inhibition of glycolysis impairs the LPS-induced differentiation of M1 macrophages. ${ }^{21)}$ M1 macrophages are predominantly pro-inflammatory immune cells that produce IL- $\beta$, TNF- $\alpha$, HMGB1, CXCL10, inducible nitric oxide synthase (iNOS), and IL-6. M2 macrophages have anti- inflammatory properties, and primarily express IL-10, IL-4, and arginase-1. The activation of M1 macrophages by LPS and interferon- $\gamma$ induces aerobic glycolysis, which involves an increased glucose uptake and the conversion of pyruvate to lactate. Lactic acid is a distinctive metabolic product of glycolysis, as oxidative phosphorylation does not produce lactic acid. The energy efficiency of glycolysis is lower than that of oxidative phosphorylation. Therefore, cells that rely on glycolysis need to consume more quantities of glucose in order to maintain energy equilibrium. The present study demonstrated that the concentrations of lactic acid in the culture medium increased after stimulation with LPS, while the concentrations of glucose decreased after stimulation with LPS. However, cotreatment with LBP suppressed the LPS-induced increase in glycolysis and the reduction in the concentrations of glucose, which suggested that LBP suppresses the LPS-induced glycolysis in macrophages.

PKM2 plays an important role in the glycolysis of cancer and immune cells. It has been reported that the expression of PKM2 is high in various types of cancer cells. ${ }^{23,24)}$ The deple- 



Fig. 5. LPS Increased PKM2 Protein via Suppressing Ubiquitination Which Was Disrupted by LBP

This study performed PCR (A) and Western blot (B) assays to assess mRNA and protein levels of Nedd4L, Nedd4, Gnb2 and Smurf1 in macrophages after treatments with LPS alone or in combination with LBP. Co-IP analysis was conducted to determine the enrichment of ubiquitin in PKM 2 protein. $* p<0.05, * * p<0.01$, and $*_{* *} p<0.01$ vs. control; ${ }^{\#} p<0.05$ and ${ }^{\# \#} p<0.01$ vs. LPS group. (Color figure can be accessed in the online version.)

tion of PKM2 diminishes the rate of glycolysis in cancer cells and also suppresses their proliferation and increases apoptosis, suggesting that the PKM2-mediated glycolysis promotes cancer progression. ${ }^{2324)}$ Xie et al. demonstrated that PKM2 promotes glycolysis in macrophages, resulting in the increased release of IL-1 $\beta$, IL-18, and HMGB1 by macrophages. ${ }^{25)}$ Ouyang et al. elucidated the critical role of PKM2 in the development of early thymocytes, including $\mathrm{CD}^{-} \mathrm{CD} 8^{-} \mathrm{T}$ cells. $^{26)}$ Deoxyelephantopin is a naturally occurring sesquiterpene lactone from Elephantopus scaber that counteracts inflammation during fulminant hepatitis by regulating PKM2 and PKM2-mediated glycolysis. ${ }^{27)}$ The results of this study demonstrated that LPS increased the expression of PKM2 mRNA by 1.3 -folds and increased the levels of PKM2 protein by 3 -folds, suggesting that the upregulation of PKM2 by LPS primarily depends on post-transcriptional strategies. It was observed that silencing PKM2 dramatically suppressed the LPS-induced glycolysis and production of pro-inflammatory factors, which suggested that PKM2 has a key role in the effects of LPS. LBP had no effects on the expression of PKM2 mRNA, however, LBP abolished the increase in the levels of PKM2 protein induced by LPS. In order to determine whether PKM2 mediated the effects of LBP against LPS-induced glycolysis and inflammation, we transfected macrophages with a PKM2 overexpression vector before treatment with LPS and LBP. LBP failed to suppress the increase in the expression of PKM2 in macrophages transfected with the PKM2 overexpression vector in the presence of LPS. Moreover, PKM2 overexpression abolished the effects of LBP against LPSinduced glycolysis and inflammation. These results suggested that LBP antagonized the effects of LPS by inhibiting the expression of PKM2.

The glycolysis induced by PKM2 induces the transcriptional transactivation of HIF-1 $\alpha$. It has been confirmed that PKM2 translocates to the nucleus, where it interacts with HIF-1 $\alpha$ and regulates the expression of numerous glycolytic enzymes by enhancing the recruitment of p300 to the HRE sites of the target genes. Moreover, PKM2 forms a complex with HIF-1 $\alpha$ and 


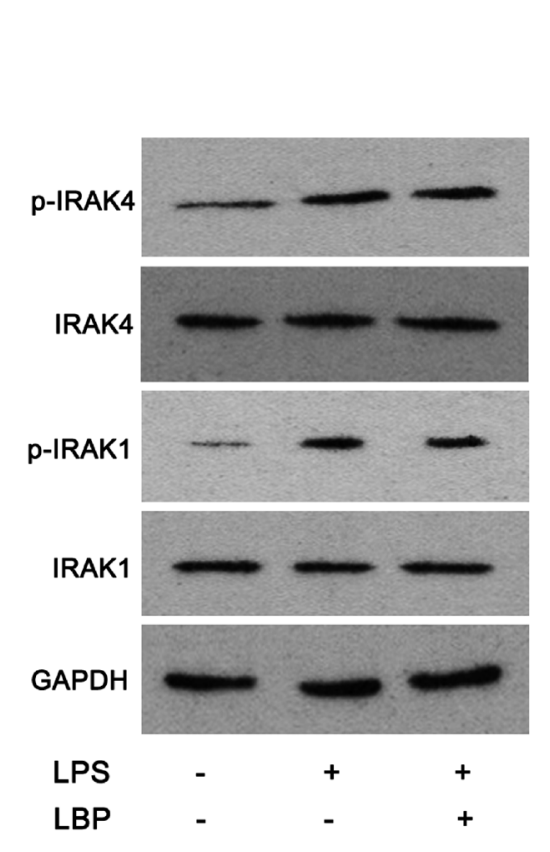

Fig. 6. LBP Had No Effect on the Activation of TLR4 by LPS
p-IRAK4
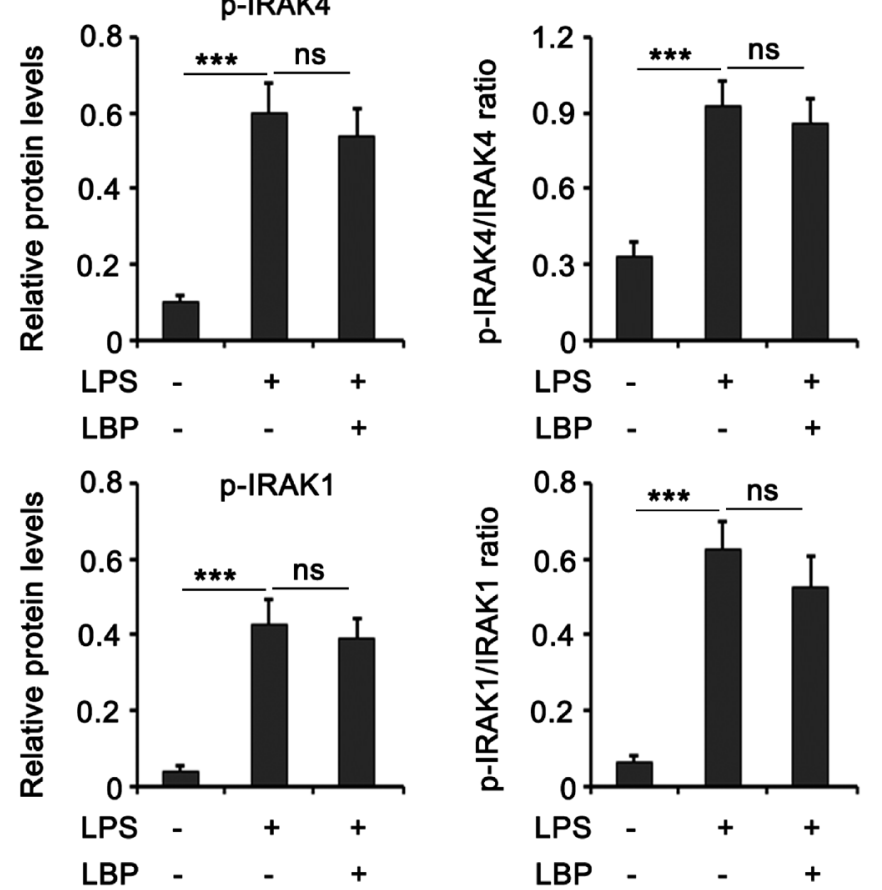

This study performed Western blot assay to assess protein levels of p-IRAK4, IRAK4, p-IRAK1 and IRAK4 in macrophages after treatments with LPS alone or in combination with LBP. $* * *<<0.01 v s$. control.

directly binds to the promoters of pro-inflammatory cytokines, and activates the transcription of IL- $1 \beta$ and HMGB1. As these pro-inflammatory cytokines are primarily secreted from M1 macrophages and seldom from M2 macrophages, this suggested PKM2 could have also affected the M1/M2 differentiation of macrophages by regulating the secretion of these pro-inflammatory cytokines. The results of this study demonstrated that LPS promoted the expression of HIF-1 $\alpha$ and its interaction with PKM2. LBP had no effects on the expression of HIF- $1 \alpha$, but suppressed the interactions between PKM2 and HIF-1 $\alpha$. This suggested that the LPS-induced differentiation of M1 macrophages was disrupted by LBP.

The results of previous data demonstrated that LPS downregulated the expression of the ubiquitin ligases, Nedd4L, Nedd4, and Gnb2, which mediate the ubiquitination of the PKM2 protein by triggering its ubiquitin-mediated degradation. These results were corroborated by the results of PCR and Western blotting. However, LBP reversed the LPSinduced downregulation of Nedd4L, Nedd4, and Gnb2, and increased the ubiquitination of the PKM2 protein. The results of this study suggested that LBP impaired the LPS-induced increase in the expression of the PKM2 protein that participates in restoring ubiquitin ligases for triggering the ubiquitination and degradation of PKM2. Zhang reported that the LPS-induced downregulation of the expression of Nedd4 is associated with the ALX/phosphatidylinositol 3-kinase (PI3K) pathway. ${ }^{28)} \mathrm{LBP}$, at least at the concentration of $50 \mu \mathrm{g} / \mathrm{mL}$, had no effect on the expression level of NEDD4, NEDD4L, and Gnb2. Therefore, LBP does not directly up-regulate NEDD4, NEDD4L, and Gnb2 to neutralize LPS-mediated inhibition in these proteins; LBP more likely disrupts LPS-induced signal that responsible for the suppression of NEDD4, NEDD4L, and Gnb2.

There is a possibility that LBP interacts with LPS directly to blocking LPS stimulating TLR4. However, this study found that LBP failed to suppress the phosphorylation of IRAK4 and IRAK1 (the up-stream effectors of TLR4 signal) after LPS stimulation. Therefore, LBP had no effect on the activation of TLR4 by LPS.

In summary, the results of this study demonstrated that LBP suppressed LPS-induced inflammation by affecting the glycolysis and the differentiation of M1 macrophages. The effects of LBP are associated with the downregulation of the expression of PKM2 mediated via the enhancement of PKM2 ubiquitination.

Acknowledgments Natural Science Foundation projects of Ningxia Autonomous Region in 2019 (No. 2019AAC03230).

Conflict of Interest The authors declare no conflict of interest

\section{REFERENCES}

1) Fleischmann C, Scherag A, Adhikari NK, Hartog CS, Tsaganos T, Schlattmann P, Angus DC, Reinhart K.; International Forum of Acute Care Trialists. Assessment of global incidence and mortality of hospital-treated sepsis. current estimates and limitations. Am. J. Respir. Crit. Care Med., 193, 259-272 (2016).

2) Kaukonen KM, Bailey M, Suzuki S, Pilcher D, Bellomo R. Mortality related to severe sepsis and septic shock among critically ill patients in Australia and New Zealand, 2000-2012. JAMA, 311, 1308-1316 (2014)

3) Napolitano LM. Sepsis 2018: definitions and guideline changes. Surg. Infect. (Larchmt), 19, 117-125 (2018).

4) Faix JD. Biomarkers of sepsis. Crit. Rev. Clin. Lab. Sci., 50, 23-36 (2013).

5) Xing L, Zhongqian L, Chunmei S, Pingfa C, Lei H, Qin J, Genhua M, Yijun D. Activation of M1 macrophages in sepsis-induced acute 
kidney injury in response to heparin-binding protein. PLOS ONE, 13, e0196423 (2018).

6) Liu YC, Zou XB, Chai YF, Yao YM. Macrophage polarization in inflammatory diseases. Int. J. Biol. Sci., 10, 520-529 (2014).

7) Cheng SC, Scicluna BP, Arts RJ, Gresnigt MS, Lachmandas E, Giamarellos-Bourboulis EJ, Kox M, Manjeri GR, Wagenaars JA, Cremer OL, Leentjens J, van der Meer AJ, van de Veerdonk FL, Bonten MJ, Schultz MJ, Willems PH, Pickkers P, Joosten LA, van der Poll T, Netea MG. Broad defects in the energy metabolism of leukocytes underlie immunoparalysis in sepsis. Nat. Immunol., 17, 406-413 (2016).

8) Kelly B, O'Neill LA. Metabolic reprogramming in macrophages and dendritic cells in innate immunity. Cell Res., 25, 771-784 (2015).

9) Tannahill GM, Curtis AM, Adamik J, et al. Succinate is an inflammatory signal that induces IL-1 $\beta$ through HIF-1 $\alpha$. Nature, 496, 238-242 (2013)

10) Cheng YY, Feng YB, Xia ZY, Li XC, Rong JH. $\omega$-Alkynyl arachidonic acid promotes anti-inflammatory macrophage M2 polarization against acute myocardial infarction via regulating the cross-talk between PKM2, HIF-1 $\alpha$ and iNOS. Biochim. Biophys. Acta Mol. Cell Biol. Lipids., 1862, 1595-1605 (2017).

11) Palsson-McDermott EM, Curtis AM, Goel G, Lauterbach MA, Sheedy FJ, Gleeson LE, van den Bosch MW, Quinn SR, DomingoFernandez R, Johnston DG, Jiang JK, Israelsen WJ, Keane J, Thomas C, Clish C, Vander Heiden M, Xavier RJ, O'Neill LA. Pyruvate kinase M2 regulates Hif-1 $\alpha$ activity and IL-1 $\beta$ induction and is a critical determinant of the warburg effect in LPS-activated macrophages. Cell Metab., 21, 65-80 (2015).

12) Yang Y, Li W, Li Y, Wang Q, Gao L, Zhao J. Dietary lycium barbarum polysaccharide induces Nrf2/ARE pathway and ameliorates insulin resistance induced by high-fat via activation of PI3K/AKT signaling. Oxid. Med. Cell. Longev., 2014, 145641 (2014).

13) Huang Y, Zhou F, Shen C, Wang H, Xiao Y. LBP reduces theinflammatory injury of kidney in septic rat and regulates the Keap1Nrf2/ARE signaling pathway1. Acta Cir. Bras., 34, e20190010000003 (2019).

14) Chen L, Li W, Qi D, Wang D. Lycium barbarum polysaccharide protects against LPS-induced ARDS by inhibiting apoptosis, oxidative stress, and inflammation in pulmonary endothelial cells. Free Radic. Res., 52, 480-490 (2018).

15) Ni H, Wang G, Xu Y, Gu X, Sun C, Li H. Lycium barbarum polysaccharide alleviates IL- $1 \beta$-evoked chondrogenic ATDC5 cell inflammatory injury through mediation of microRNA-124. Artif Cells Nanomed Biotechnol., 47, 4046-4052 (2019).

16) Liu S, Xiao Z, Ai F, Liu F, Chen X, Cao K, Ren W, Zhang X, Shu
P, Zhang D. miR-142-5p promotes development of colorectal cancer through targeting SDHB and facilitating generation of aerobic glycolysis. Biomed. Pharmacother., 92, 1119-1127 (2017).

17) Tallam A, Perumal TM, Antony PM, Jäger C, Fritz JV, Vallar L, Balling R, Del Sol A, Michelucci A. Gene regulatory network inference of immunoresponsive gene 1 (IRG1) identifies interferon regulatory factor 1 (IRF1) as its transcriptional regulator in mammalian macrophages. PLOS ONE, 11, e0149050 (2016).

18) Lu YC, Yeh WC, Ohashi PS. LPS/TLR4 signal transduction pathway. Cytokine, 42, 145-151 (2008).

19) Zhong WJ, Yang HH, Guan XX, Xiong JB, Sun CC, Zhang CY, Luo XQ, Zhang YF, Zhang J, Duan JX, Zhou Y, Guan CX. Inhibition of glycolysis alleviates lipopolysaccharide-induced acute lung injury in a mouse model. J. Cell. Physiol., 234, 4641-4654 (2019).

20) Galván-Peña S, O’Neill LAJ. Metabolic reprograming in macrophage polarization. Front. Immunol., 5, 420 (2014).

21) Wang F, Zhang S, Jeon R, Vuckovic I, Jiang X, Lerman A, Folmes CD, Dzeja PD, Herrmann J. Interferon gamma induces reversible metabolic reprogramming of M1 macrophages to sustain cell viability and pro-inflammatory activity. EBioMedicine, 30, 303-316 (2018).

22) Liu PS, Ho PC. Determining macrophage polarization upon metabolic perturbation. Methods Mol. Biol., 1862, 173-186 (2019).

23) Liu F, Ma F, Wang Y, Hao L, Zeng H, Jia C, Wang Y, Liu P, Ong IM, Li B, Chen G, Jiang J, Gong S, Li L, Xu W. PKM2 methylation by CARM1 activates aerobic glycolysis to promote tumorigenesis. Nat. Cell Biol., 19, 1358-1370 (2017).

24) Li YH, Li XF, Liu JT, Wang H, Fan LL, Li J, Sun GP. PKM2, a potential target for regulating cancer. Gene, 668, 48-53 (2018).

25) Xie M, Yu Y, Kang R, Zhu S, Yang L, Zeng L, Sun X, Yang M, Billiar TR, Wang H, Cao L, Jiang J, Tang D. PKM2-dependent glycolysis promotes NLRP3 and AIM2 inflammasome activation. Nat. Commun., 7, 13280 (2016).

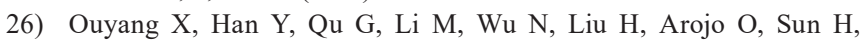
Liu X, Liu D, Chen L, Zou Q, Su B. Metabolic regulation of T cell development by Sin1-mTORC2 is mediated by pyruvate kinase M2. J. Mol. Cell Biol., 11, 93-106 (2019).

27) Pan L, Hu L, Zhang L, Xu H, Chen Y, Bian Q, Zhu A, Wu H. Deoxyelephantopin decreases the release of inflammatory cytokines in macrophage associated with attenuation of aerobic glycolysis via modulation of PKM2. Int. Immunopharmacol., 79, 106048 (2020).

28) Zhang JL, Zhuo XJ, Lin J, Luo LC, Ying WY, Xie X, Zhang HW, Yang JX, Li D, Gao Smith F, Jin SW. Maresin1 stimulates alveolar fluid clearance through the alveolar epithelial sodium channel $\mathrm{Na}$, K-ATPase via the ALX/PI3K/Nedd4-2 pathway. Lab. Invest., 97, 543-554 (2017). 